\title{
ANÁLISIS DEL APOYO ACADÉMICO FAMILIAR EN ESTUDIANTES DE EDUCACIÓN BÁSICA SECUNDARIA EN INSTITUCIONES EDUCATIVAS DEL MUNICIPIO DE TULUÁ EN COLOMBIA
}

\author{
Reinaldo Castrillón Mosquera \\ Universidad Internacional Iberoameriana (UNINI). Campeche, México. \\ Manuel Soriano Ferrer \\ Universidad de Valencia. Valencia, España.
}

\begin{abstract}
Resumen. La presente investigación tiene como objetivo analizar el apoyo académico parental que reciben alumnos de educación básica secundaria de instituciones educativas públicas ubicadas en Tuluá Colombia con la finalidad de determinar si existen diferencias entre el apoyo que reciben alumnos con buen rendimiento académico y el apoyo que reciben alumnos con bajo rendimiento. En la investigación, se usó metodología descriptiva sobre una muestra de 608 estudiantes (304 hombres y 304 mujeres) con edades entre 11 y 16 años matriculados en los grados $6^{\circ}, 7^{\circ}, 8^{\circ}$ y $9^{\circ}$ de educación secundaria, teniendo en cuenta para la selección los resultados obtenidos en el primer período escolar del año 2016 comprendido entre los meses de enero a marzo. Para la recolección de los datos se aplicó escala tipo Likert compuesta por 35 ítems buscando medir el nivel de percepción de los estudiantes en cuanto al apoyo brindado por los padres en aspectos como acompañamiento en la realización de tareas, comunicación con el centro escolar, fijación de normas, motivación e interés a las situaciones propias del entorno. El 97\% de los estudiantes segmentados en bajo rendimiento considera que si los padres se involucraran más en los aspectos escolares, su desempeño académico sería mejor. Yuxtapuesto a ello, el 100\% de los estudiantes con buen rendimiento percibe como fundamental para los logros obtenidos el apoyo paternal recibido. El estudio permite concluir que el apoyo académico familiar en cabeza de los padres, influye de forma positiva y directa en el rendimiento de estudiantes de educación básica secundaria.
\end{abstract}

Palabras clave: rendimiento académico, apoyo académico parental, entorno escolar, tareas para la casa, contexto familiar.

\section{ACADEMIC FAMILIAR SUPPORT ANALYSIS OF MIDDLE SCHOOL STUDENTS IN EDUCATIONAL INSTITUTIONS FROM TULUÁ, COLOMBIA.}

\footnotetext{
Abstract. This research aims to analyze the academic and familiar support that some middle school students receive in educational institutions located in Tuluá, Colombia, in order to determine whether there are differences between the support for students with good academic performance and the support for students with low performance. For this research, a descriptive methodology was employed using 608 students as sample (304 men and 304 women) aged between 11 and 16 years enrolled in 6th, 7th, 8th and
} 
9th grade of high school, taking also into account the academic results obtained during the last academic year. For collecting the data, a Likert scale was applied composed by 35 items, which it was intended to measure the students perception in the academic support provided by parents in some aspects like support in fulfilling tasks, communication with the school center, standard setting, motivation and interest to the specific situations of the environment. $97 \%$ of students segmented in the low performance group believe that, if their parents became more involved in school issues, the academic performance would be better. Juxtaposed to this, $100 \%$ of the students with good performance perceives as fundamental they received parental support for their achievement. The study allows us to conclude that family academic support being led by the parents influences positively and directly in the academic performance of students in high school.

Keywords: academic achievement, family academic support, school environment, homework, family context.

\title{
ANÁLISE DO APOIO ACADÊMICO FAMILIAR EM ESTUDANTES DO ENSINO FUNDAMENTAL SEDUNDÁRIO EM INSTITUIÇÕES EDUCACIONAIS DO MUNICÍPIO TULUÁ - COLÔMBIA
}

\begin{abstract}
Resumo. Esta pesquisa tem como objetivo analisar o apoio académico da familia aos estudantes do Ensino Fundamental secundário de instituições públicas, situadas em Tuluá - Colômbia, a fim de determinar se existem diferenças entre o apoio aos estudantes com melhor desempenho em relação aos com baixo desempenho. Optou-se pela pesquisa descritiva, envolvendo uma amostra de 608 alunos (304 do sexo masculino e 304 do sexo feminino), com idades entre 11 e 16 anos, matriculados no $6^{\circ}, 7^{\circ}, 8^{\circ}$ e $9^{\circ}$ do Ensino Fundamental secundário, considerando, na seleção, os resultados obtidos no primeiro período escolar do ano de 2016, compreendido entre os meses de janeiro e março. Para a coleta de dados foi aplicada uma escala tipo Liker, composta por 35 itens, buscando medir o nível de percepção dos estudantes em relação ao apoio proporcionado pelos pais em questões como a realização de tarefas, comunicação com a escola, estabelecimento de normas, motivação e interesse para as situações próprias do entorno. Os resultados indicam que $97 \%$ dos estudantes do grupo alvo de baixo desempenho consideram que se os pais se envolvessem mais nas questões escolares o desempenho seria melhor. Em contrapartida, $100 \%$ dos alunos com bom desempenho consideram como fundamental o apoio recebido da família para os resultados alcançados. $\mathrm{O}$ estudo permite concluir que a família, sob a condução dos pais, influencia positivamente e diretamente no desempenho escolar dos estudantes do Ensino Fundamental secundário.
\end{abstract}

Palavras-chave: desempenho acadêmico, apoio acadêmico famíliar, ambiente escolar, trabalhos de casa, contexto familiar. 


\section{Introducción}

Los resultados académicos en la educación básica secundaria son un tema preocupante en Colombia, rasgo obtenido de las mediciones nacionales e internacionales que se realizan en el país. Numerosas investigaciones convergen en las conclusiones en la incidencia que el contexto familiar tiene en el rendimiento académico de los estudiantes, especialmente en los aspectos relacionados al apoyo brindado por los padres al proceso de formación de los menores. La familia es el núcleo básico de la sociedad. De este núcleo o grupo organizado en las formas tradicionales de composición como un padre, una madre y los hijos se generan los ciudadanos que le han dado forma al esquema social, tanto en el pasado como en el presente. Hoy en día, la composición familiar no es tan tradicional ya que se observa que hay una dinámica fuerte de cambio en el enfoque inicial, surgiendo de esta forma conceptos diversos a lo que se percibe como familia.

Gutiérrez, Camacho y Martínez (2007) exponen que la familia es el grupo natural primario en el cual los padres garantizan y protegen el desarrollo psicosocial de los miembros, transmitiendo de igual modo la cultura para que los integrantes generen valores que los lleven a tener una autoestima alta y autorrealización. En ese sentido, Bas y Pérez (2010) argumentan que la familia es el motor central en la vida de las personas, siendo necesario definir relaciones entre la familia y el profesorado que proporcionen a los estudiantes una educación crítica y los ubique en el entorno de forma reflexiva y constructiva, dando lugar a ciudadanos formados a partir de la vivencia de valores y del soporte familiar que otorgan sentido de persona y de ciudadanía. Conjugar este tipo de apoyo, acerca la realidad familiar del niño y adolescente con su realidad escolar formando un todo indivisible que favorece el aprendizaje. Si tanto padres como docentes se integran en el proceso se completa la base necesaria para obtener buenos resultados en la preparación académica y personal del estudiante.

Enríquez, Segura y Tovar (2013) manifiestan que en la etapa de la adolescencia, cuando el joven está cursando la educación secundaria está atento y dispuesto a lo que el entorno le ofrece. Por lo cual, si el entorno sea familiar o social le ofrece situaciones positivas, así mismo serán los resultados en los proyectos que esté viviendo, entre ellos el escolar. Por el contrario, si del contexto percibe situaciones negativas, fácilmente puede caer en ellas como la violencia, la drogadicción, la soledad, la antipatía e indiferencia por el estudio, la depresión y demás fenómenos sociales latentes. Erazo (2010) considera que la adolescencia y la etapa escolar secundaria son momentos decisivos de acompañamiento y apoyo familiar porque de ello depende el futuro académico del estudiante.

Para Faires, Nichols y Rickelman (2000) dentro del acompañamiento que la familia debe otorgarle a los hijos a nivel académico es preciso que coincidan tres actividades, en conjunto con las demás acciones que cada padre considere para brindar apoyo al niño. Las tres actividades a coexistir con las cuales se proyecta buen 
rendimiento académico en el estudiante son: a) acompañar al niño de forma significativa en la realización de las tareas; b) fijar un tiempo y espacio dentro de la casa para el estudio; c) comunicarse de forma constante con los maestros del estudiante.

Hoover-Dempsey (2001) refiere que el apoyo académico familiar no debe limitarse solamente al acompañamiento en la realización de las tareas escolares; esta actividad debe extenderse a aspectos como revisión y seguimiento de las mismas una vez hayan sido calificadas, repaso de los conceptos vistos en clase, dedicación de tiempo a lecturas complementarias, entre otras acciones con las que se optimice el apoyo. En este sentido, el autor refiere cuatro razones por la cual los padres deben estar al tanto del progreso académico de los hijos, acompañándolos permanentemente en el rol de estudiante: 1) las expectativas que como padres tengan respecto a la educación que están recibiendo los hijos, enfocada en el futuro mismo de los niños. 2) la convicción de que al ayudarlos, el desempeño académico será mejor. 3) el aumento en la competencia académica de los padres al ayudar a los hijos, por lo cual es pertinente decir que en un proceso de aprendizaje quien aprende es quien enseña. 4) la percepción positiva que tendrá el niño de los padres al observar el apoyo continuo de parte de ellos.

Luego de realizar investigación a estudiantes de instituciones públicas de Guatemala, Porta y Laguna (2008) concluyen que una de las revoluciones que en materia educativa deben darse, es precisamente la inclusión permanente de las familias en actividades escolares que conlleven a optimizar una cercanía de los padres con los centros educativos fomentando de esta forma la integración necesaria para que desde las casas se apoye la labor de los docentes, lo que dará resultados positivos en el rendimiento académico de los jóvenes.

En análisis de Desforges y Abouchaar (2003) a diversas investigaciones realizadas sobre el impacto de la implicación parental, el apoyo académico familiar y el nivel de educación de los padres en el rendimiento escolar de los estudiantes tomando como referencia estudios realizados en Norteamérica, Australia, Europa, Escandinavia y el Reino Unido en cuyo objetivo se tenía la determinación de variables familiares que presentaran influencia en el rendimiento escolar, se registra como conclusión general que la implicación parental influencia positivamente el rendimiento de los alumnos indistintamente en la etapa escolar en la que se encuentre, enmarcando un buen apoyo en las siguientes características: las actividades escolares que se realizan en conjunto entre padres e hijos otorgan seguridad al estudiante y afianzan su auto concepto; las expectativas de los padres en cuanto al proceso escolar de los hijos son la base para las metas personales de los jóvenes; tener comunicación permanente con los docentes, con la escuela y conocer las normas de la institución, crea un clima de cercanía entre los estudiantes, los padres y el contexto escolar; visitar la escuela, participar de forma directa en los eventos que programen, sugerir mejoramientos, hacer parte de los órganos de administración y gobierno escolar y supervisar las actividades deportivas son estrategias parentales en las que el estudiante percibe importancia en lo que realiza, trascendiendo el sentir en los logros y en el rendimiento académico. 
Núñez, Suárez, Rosário, Vallejo, Valle y Epstein (2015) presentan resultados de investigación sobre la relación entre la implicación parental en la realización de las tareas y el comportamiento de los estudiantes a la hora de afrontar los deberes escolares con el rendimiento académico; analizando las diferencias encontradas en los grupos objeto del estudio como lo fueron escuela elemental, básica secundaria y grados superiores. La muestra estuvo conformada por 1.683 estudiantes españoles con un rango de edades comprendidas entre 10 y 16 años pertenecientes a 10 colegios públicos urbanos quienes participaron en la investigación de forma voluntaria con la aprobación previa de los padres, aplicándoles una escala tipo Likert de 1 a 5 en la que 1 era "completamente en desacuerdo" y 5 "completamente de acuerdo", con 13 frases en su mayoría afirmativas estructuradas en tres componentes: control, apoyo y normas o conductas en casa para la elaboración de tareas. Los datos, manejados estadísticamente con el programa Mplus 5.1, permitieron concluir que existe una estrecha relación entre el apoyo académico familiar que los padres brindan a los hijos en la realización de las tareas, además de las conductas adoptadas por ellos en el desarrollo de las mismas con el rendimiento académico obtenido en el proceso de aprendizaje; cumpliéndose la hipótesis planteada al inicio de la investigación.

El contexto familiar en el rendimiento académico es un tema que ha sido diversamente estudiado en los últimos años. Prueba de ello es investigación realizada por Caicedo y Cepeda (2007) en la que por medio de la aplicación de 1.750 encuestas a igual número de estudiantes de colegios ubicados en Bogotá en las cuales se les consultó aspectos familiares, escolares y de los medios de comunicación que pudieran llegar a afectar el rendimiento académico se concluye que algunos de los factores que inciden negativamente en el desempeño colegial de los adolescentes son la indiferencia y la falta de diálogo entre padres e hijos sobre las experiencias que se viven en la escuela. Paralelo a ello se determinan como factores familiares y escolares que favorecen el rendimiento académico los relacionados a un buen ambiente familiar, el apoyo constante de los padres en la elaboración de trabajos y tareas y el acompañamiento permanente en las actividades propias de la escuela generando una motivación mayor de los estudiantes en el ámbito escolar.

En investigación de Hernando, Oliva y Pertegal (2012) a estudio realizado sobre una muestra de 2.400 estudiantes entre 12 y 17 años adscritos a 20 centros educativos de Andalucía en España, se analizó la correlación existente entre el rendimiento académico de los adolescentes seleccionados y variables como el tiempo dedicado a la realización de los deberes escolares, el estatus socioeconómico de las familias, el estilo educativo de los padres; así como el desarrollo evolutivo y el género de los adolescentes. Se concluye en el estudio que las correlaciones más altas en cuanto al rendimiento se presentan entre el tiempo dedicado a la realización de las tareas y el nivel de estudio de los padres y lo que menos está relacionado con el rendimiento escolar es el componente de humor que perciba el estudiante de la familia. 
Para Giordano (2003) de una buena relación entre los adolescentes y la familia depende el sano desarrollo de la personalidad, máxime en esa etapa del crecimiento donde sentir el apoyo y el acompañamiento de alguien es para el estudiante un punto de partida hacia la vida adulta, configurándose de este modo personas seguras de sí mismas, sin temores y con proyección hacia el éxito y no hacia el fracaso. En investigación de Iraurgi, Martínez, Iriarte y Sanz (2011) a una muestra conformada por 3.957 estudiantes de educación secundaria obligatoria de 36 centros educativos de Biskaia cuyo objetivo era medir la relación de los conflictos interparentales con el nivel de adaptación del adolescente en diferentes espacios y la influencia de los mismos en el rendimiento académico se pudo concluir que los alumnos con niveles altos de fracaso escolar son aquellos procedentes de hogares con baja afectividad por parte de los padres, presentando constantemente conflictos al interior de la familia.

Conclusiones similares registran Peralta y Espinoza (2009) luego de estudio realizado a una muestra conformada por 56 alumnos de educación básica de Cuenca en Ecuador, padres de familia, profesores y personal administrativo de una institución educativa cuyo enfoque era relacionar la incidencia de la violencia intrafamiliar en el desarrollo y rendimiento académico de los estudiantes, encontrando que en un $8 \%$ de alumnos segmentados en resultados de dificultad en el proceso, primaba el desinterés familiar, poco apoyo por parte de los padres y violencia intrafamiliar física y psicológica al interior de los hogares. Contrario a ello, los estudiantes con buen rendimiento académico, $92 \%$ de los encuestados, presentaban características de afecto, bajos índices de violencia intrafamiliar y apoyo constante de los padres en la escolaridad de los hijos.

Bodensiek (2010) luego de investigación realizada para la Secretaría de Educación de Bogotá cuyo objetivo principal era estudiar los factores que determinaran el rendimiento académico de estudiantes matriculados en instituciones educativas públicas y privadas concluye que factores como la edad y el género de los alumnos, composición y características familiares, nivel educativo y profesional de los padres, ambiente familiar, prácticas de crianza, comunicación de los padres con la escuela y con el entorno de aprendizaje de los hijos, entre otros; son factores que se relacionan directamente con el rendimiento escolar.

Risso, Peralbo y Barca (2010) al investigar a una muestra conformada por 1.392 estudiantes de educación secundaria obligatoria de Galicia, España con edad promedio de 14 años, concluye que el contexto familiar es determinante en el rendimiento académico de los adolescentes. Para ello se midieron y analizaron variables como la composición familiar, nivel de estudio de los padres, condiciones del estudio en casa, estabilidad emocional de los miembros del hogar, valoración positiva de los logros, entre otros aspectos encaminados a la búsqueda de los factores que desde el hogar contribuyen al desempeño positivo estudiantil. Para Schlee, Mullis y Shriner (2009) el contexto familiar condiciona la posición de salida de los estudiantes e incide directamente en la autorregulación y el curso del aprendizaje escolar. 
Con el objetivo general de establecer la relación de factores personales, familiares y académicos que son determinantes en el fracaso escolar en la escuela secundaria, Lozano (2003) llevó a cabo investigación a una muestra conformada por 1.178 estudiantes de Almería, cursantes de los cuatro grados en que se compone la educación secundaria obligatoria conocida en España con la sigla ESO. En la investigación se plantean cuatro hipótesis que de forma general buscan encontrar evidencias de que diferentes variables del contexto del estudiante y del propio comportamiento influyen de forma directa en el rendimiento. En el conjunto de hipótesis se toca de forma explícita las relaciones de padres hacia hijos, el apoyo académico parental y el nivel de estudio de los padres como influyentes en el rendimiento o en el fracaso escolar dependiendo la medida en la que estén presentes. Los resultados de la investigación permiten concluir que las variables de tipo familiar están relacionadas de forma directa con el rendimiento de los estudiantes, encontrando que variables como el nivel de estudio del padre jalona una influencia positiva en los logros escolares de los hijos y en conjunto con el nivel de estudios de la madre son bases para determinar una adecuada motivación académica en el educando, siendo esta variable en orden de importancia la que ocupa el primer lugar conforme a las conclusiones referidas.

En investigación de Xu y Corno (2006) sobre la influencia del apoyo académico parental específicamente en lo relacionado a la gestión de las tareas escolares y cómo este apoyo se ve reflejado en el rendimiento de los estudiantes concluyen que la incidencia de las variables familiares es alta dejando efectos positivos en los casos donde la participación parental es significativa. Para el estudio conformaron una muestra de 238 alumnos de grados $7^{\circ}$ y $8^{\circ}$ de una escuela rural de secundaria de Tennessee, con economía familiar basada en manufactura, trabajo en fábricas y servicios industriales. Uno de los reactivos contenidos en la encuesta estaba destinado a preguntarles si eran acompañados por los padres en la realización de las tareas. El 69\% de los consultados respondió afirmativamente el interrogante planteado.

El estudio permite concluir que los estudiantes que reportaron ayuda de los padres al momento de realizar las tareas en comparación con los estudiantes que no reciben ayuda ni acompañamiento familiar, gestionan de forma más asertiva los deberes escolares, tienen mayor automotivación en el proceso académico, controlan adecuadamente las emociones lo que influye directamente en el rendimiento. La investigación demuestra que el porcentaje de estudiantes en los cuales los padres no los apoyan en las situaciones escolares presentan un bajo rendimiento y poca motivación hacia el logro de resultados, falta de expectativa en las vivencias como estudiante y orientación al fracaso.

Dumont, Trautwein, Nagy y Nagengast (2013) llevaron a cabo estudio en Alemania sobre el apoyo parental en la realización de tareas escolares en una muestra conformada por 2.830 adolescentes de grados $5^{\circ}$ y $7^{\circ}$ encuestando de igual modo a los padres de los estudiantes. En el trabajo de campo se dio prelación al análisis de 
variables que indicaran la calidad de participación de los padres en los deberes escolares y la influencia que generaban en el rendimiento, principalmente en la comprensión lectora. La escala se agrupó en torno a tres componentes: el control de los padres, la capacidad de respuesta y la formación académica. Al analizar los resultados, los autores concluyen que el contexto socioeconómico de las familias no deterioraba el nivel de apoyo que se les podría brindar a los hijos. De igual modo, concluyen que la formación académica de los padres prestó mayor importancia en el apoyo de los estudiantes de grado séptimo que en los de grado quinto, pero había mayor control de los padres en la ejecución de los deberes de los estudiantes de quinto que en los de séptimo.

En investigación de Waqas, Fatima, Sohail, Saleem y Khan (2013) a una muestra de 375 estudiantes hombres y mujeres de grado noveno de educación básica secundaria de la ciudad de Lahore en Pakistán cuyo objetivo principal era explorar los efectos del apoyo parental en el rendimiento académico de los hijos se pudo comprobar con los resultados las hipótesis planteadas, encontrando alta significancia en la correlación de las variables estudiadas. En el análisis realizado se argumenta que la educación es la forma esencial del desarrollo de las sociedades y entre mayor número de personas se eduquen el desarrollo se logra con mayor rapidez. Bajo este esquema, las familias son las principales responsables de que los hijos sean miembros productivos para la sociedad y esto se logra en la medida que los padres se involucren en el proceso educativo conllevando a que los niños en el futuro tengan excelentes carreras académicas y desempeños laborales exitosos.

Como hipótesis de la investigación pakistaní se planteó: a mayor involucramiento de los padres en las actividades académicas de los hijos, mayor será su rendimiento escolar. Yuxtapuesto a ello la hipótesis nula estuvo enmarcada en el efecto contrario donde no había ninguna relación entre el apoyo académico de los padres y el rendimiento de los estudiantes. Los hallazgos del estudio permitieron definir las siguientes conclusiones: a) hay una alta relación entre el nivel de participación de los padres en las actividades académicas de los hijos y el nivel del rendimiento logrado por ellos en la escuela y b) el estudio valida los seis tipos de involucramiento definidos por Sptein en 1995 que afectan de forma positiva el desempeño escolar: apoyo, comunicación, cercanía de los padres con el centro escolar, aprendizaje en casa, toma de decisiones y colaboración con la comunidad.

En estudio de Núñez, Vallejo, Rosário, Tuero y Valle (2014) en la que se analizaron variables relacionadas al estudiante y variables relacionadas al contexto se investigó la incidencia que tenían en el rendimiento escolar. Para ello se conformó una muestra de 988 estudiantes de último año escolar en conjunto con 57 profesores de biología, área definida para evaluar el desempeño. Los alumnos pertenecían a 10 escuelas de Portugal contando con la autorización previa de los padres y de los docentes para llevar a cabo las mediciones en los menores. De los 988 estudiantes, 384 eran hombres y 604 mujeres con un rango de edades comprendido entre 16 y 19 años. La 
experiencia de los docentes estaba comprendida entre 2 y 36 años de carrera en el sector educativo.

Las variables directamente relacionadas al estudiante estaban definidas por rendimiento académico, tiempo dedicado al estudio, conocimientos previos en biología, ausentismos de clase y la realización de tareas. Como variables de clase se tomaron en cuenta aspectos relacionados al nivel de conocimiento, experiencia y género de los profesores; mientras que para las variables del contexto se indagó por el tamaño del grupo y el nivel de educación de los padres. Con una perspectiva multinivel se analizaron los datos, concluyendo de los mismos que el rendimiento de los estudiantes en el área observada depende en un $85,6 \%$ de las variables propias del estudiante, mientras que del contexto y de las variables de clase depende el porcentaje restante $14,4 \%$, haciendo precisión en la incidencia que tiene el nivel educativo de los padres en el desempeño escolar de los estudiantes.

Oubrayrie-Roussel y Safont-Mottay (2011) refieren que en la etapa de la adolescencia, la escuela, los compañeros de clase y la familia llegan a tener alta influencia en el desarrollo psicológico y en el avance escolar. Para comprobar la hipótesis llevaron a cabo investigación en la cual colocaron como eje central la importancia de la familia y la influencia del apoyo parental en el desarrollo de la autonomía y de habilidades de los jóvenes a nivel personal y escolar. Basándose en el modelo de involucramiento parental propuesto por Epstein, el estudio tiene como preguntas de investigación las siguientes: ¿Cómo los adolescentes perciben el apoyo de los padres? ¿El apoyo de los padres es determinante para el desarrollo de la autonomía y del rendimiento académico de los hijos?

La muestra conformada por 504 adolescentes franceses matriculados en instituciones de carácter público de edades comprendidas entre 12 y 15 años se caracterizó por tener 244 hombres y 260 mujeres, seleccionados de diferentes grados de educación básica secundaria. Para analizar el apoyo parental se definieron tres escalas con cinco opciones de respuesta donde 1 era el nivel más bajo o nunca y 5 el nivel más alto o siempre. En la primera escala se le dio importancia a la medición de la percepción del estudiante en cuanto al apoyo que observaba de parte de los padres en las tareas escolares midiendo situaciones como involucramiento de los adultos en la realización de las tareas, orientación, seguimiento; así como cerciorarse de que les haya quedado bien hecha. La segunda escala se centró en indagar sobre el nivel de percepción que tenían los estudiantes referentes a la efectividad, habilidades y conocimiento de los padres al momento que les brindaban ayuda en la elaboración de las tareas; escala que se complementaba con la tercera en la que se daba precisión a las estrategias parentales empleadas para dar una debida terminación de las tareas y la concentración de los alumnos al momento de realizarlas. Por lo cual, en esta escala se tomaron en cuenta aspectos encaminados a disminuir las distracciones, manejo de tiempos, definición de horarios, adecuación de espacios, seguimiento y control de las emociones. 
En las conclusiones del estudio, se registra que la percepción que tienen los adolescentes en cuanto a la eficacia de los padres en el apoyo que les brindan a nivel escolar tienen efectos directos en la auto regulación, autonomía y en el desempeño conllevando a que se tomen decisiones acertadas en los proyectos a corto, mediano y largo plazo.

En investigación de Chen (2008) en la que se midió la percepción que tenían estudiantes de educación secundaria sobre el apoyo brindado por los padres, los profesores y los compañeros y la influencia que ese apoyo pudiera tener en el rendimiento, se puede concluir que la implicación parental y de los docentes es influyente en el progreso escolar de los estudiantes teniendo en cuenta para ello condiciones culturales, educacionales, socioeconómicas y perspectivas de desarrollo del contexto en el que se desenvuelve el adolescente. Para el estudio se conformó una muestra de 270 alumnos de educación secundaria de Hong Kong de los grados $3^{\circ}$ a $5^{\circ}$, equivalentes a los grados $9^{\circ}$ a $11^{\circ}$ de los Estados Unidos concluyendo con los resultados que la implicación parental es de mayor significación en el rendimiento académico en el grado tercero que en el grado cuarto, al igual que el apoyo por parte de los profesores; el cual los alumnos encuestados lo perciben con mayor influencia en los cursos inferiores que en los superiores. Complementario a ello la relación entre compañeros no es determinante del rendimiento, teniendo la convivencia escolar mayor efecto en aspectos de tipo social que de tipo académico.

Erazo (2010) realizó investigación de tipo descriptivo-cualitativo con la que buscaba describir los problemas que afectaban el rendimiento académico de los estudiantes de educación básica secundaria de nueve instituciones educativas de carácter público en Popayán-Colombia. Para ello aplicó instrumentos de escala, entrevista y observación directa a una muestra de 487 participantes distribuida en 187 estudiantes, 88 docentes, 203 padres de familia y 9 directivos docentes; dividiendo la investigación en cuatro componentes de focalización: acciones y dificultades del centro escolar, situaciones problema del estudiante con los compañeros y con los docentes, problemas del estudiante con el contexto social y problemas del estudiante en torno a la familia, la drogadicción y la sexualidad. Popayán es una ciudad que por las características físicas, entorno habitacional, educativo y social se asimila al contexto de la ciudad de Tuluá, lugar donde se lleva a cabo el presente estudio.

En los estudiantes que presentan bajo rendimiento escolar, los resultados obtenidos permiten concluir que: a) Hay escasa o nula comunicación entre padres e hijos; así como se percibe poca comunicación entre los mismos padres, de los padres con los docentes y con la escuela en general. b) La solución a los problemas familiares no se hace de forma concertada, primando el autoritarismo de uno de los padres al momento de tomar las decisiones. c) La conformación de las familias no es la tradicional. Hay hogares donde los padres se han separado y los estudiantes viven ya sea con el papá, la mamá y en diversos casos con los abuelos, lo que provoca situaciones de aislamiento y de ansiedad en el alumno. d) Existe carencia de normas en cuanto al 
cumplimiento de los deberes escolares, carencia de autoridad en los padres aplicando verticalidad y autoritarismo en el contex to familiar. e) Los padres no comparten tiempo con los hijos en actividades encaminadas al sano esparcimiento ni a la enseñanza de experiencias personales. No se comparte con los hijos la realización de las tareas o actividades académicas. f) La responsabilidad de crianza de los hijos se transfiere a la escuela y a los docentes mostrando despreocupación e indiferencia por las vivencias de los estudiantes en el centro escolar.

Las diferentes conclusiones de los estudios referidos dan cuenta de la relación existente entre un buen rendimiento escolar y variables de tipo familiar tales como armonía y estabilidad del hogar, composición tradicional, empleabilidad, nivel de educación de los padres, acompañamiento y apoyo parental en el proceso educativo; al igual que la motivación que desde el hogar se promueva hacia el estudio. Contrario a ello, entre las variables familiares que desfavorecen el rendimiento escolar se registran falta de acompañamiento de los padres en el proceso, violencia intrafamiliar, desempleo, poca o nula formación académica parental, irrespeto hacia los hijos, desmotivación académica; conllevando el conjunto de factores a pobres resultados en la formación integral y en el aprendizaje del educando.

\section{Método}

En la presente investigación con metodología descriptiva y enfoque cuantitativo se proyecta como objetivo general: analizar, desde la perspectiva misma de los educandos objeto del estudio, el apoyo académico familiar que reciben alumnos de educación básica secundaria de instituciones educativas públicas en Tuluá Colombia; por parte del investigador quien se desempeña como coordinador educativo del municipio, con la finalidad de determinar si existen diferencias entre el apoyo académico familiar percibido por alumnos con buen desempeño académico y la percepción del apoyo por parte de estudiantes con bajo rendimiento.

Dentro de los objetivos específicos se proyectan los siguientes: a) Referir a partir del análisis de los resultados de la investigación, si el acompañamiento que los padres proporcionan en el proceso educativo de los hijos puede llegar a influir en la obtención de un buen rendimiento académico de los mismos y si por el contrario la falta de apoyo parental en el proceso es causa de bajo desempeño escolar. b) Comparar si el grado de refuerzo que los alumnos reciben de los padres al momento de llevar a cabo actividades en conjunto como la realización de tareas en casa y la competencia de los padres en el desarrollo de las mismas, difiere entre alumnos con buen desempeño académico y alumnos con bajo desempeño académico. c) Indagar a los alumnos objeto de la investigación sobre la percepción que tienen del apoyo que reciben de los padres, analizando el grado de diferencia entre la percepción que tienen los estudiantes con buen desempeño académico y la percepción que tienen los estudiantes con bajo desempeño, estableciendo las diferencias entre uno y otro grupo. 
Tanto el objetivo general como los específicos guardan estrecha relación con la hipótesis de investigación en la cual partiendo de la muestra objeto del estudio, se espera encontrar diferencias significativas entre el nivel de apoyo académico parental que reciben alumnos con buen desempeño académico y el que reciben alumnos con bajo desempeño. En concreto, se espera que los estudiantes con bajo desempeño académico reciban menos apoyo de los padres que los estudiantes con buen desempeño.

Para delimitar el campo fue necesario ubicarse en instituciones educativas oficiales del municipio de Tuluá, Colombia donde se llevó a cabo la investigación, la cual se enfoca en adolescentes de educación básica secundaria de ambos géneros, a quienes se aplicaron los instrumentos definidos para el estudio. En observación realizada por el investigador al entorno de las instituciones educativas seleccionadas como población objeto y de las fichas de caracterización contenidas en las matrículas de los estudiantes conformantes de la muestra se obtiene información que permite referir que la caracterización familiar corresponde a estratos socioeconómicos bajos, con escasos recursos monetarios, carencia de empleo, riesgo de adicción a las drogas, vulnerabilidad a violencia intrafamiliar; previendo que los alumnos llegan cargados de sentimientos negativos a las aulas por la realidad que viven en las casas. No obstante, se percibe que son jóvenes que le apuestan de forma positiva a la preparación académica como punto de partida para un progreso a futuro.

En la búsqueda de la ejecución de los objetivos general y específicos, la población objeto del estudio corresponde a 1.820 estudiantes de seis instituciones educativas de carácter oficial del municipio de Tuluá en Colombia con un rango de edades entre los 11 y los 16 años de edad, cursando grados académicos de sexto a noveno de educación básica secundaria. Para el cálculo de la muestra se usó herramienta de la Red de Bibliotecas UNNE (2016), estimando como margen de error un 3\% y un nivel de confianza de $93 \%$, quedando conformada por 608 estudiantes, bajo la técnica de muestreo aleatorio simple. Igual mecanismo usado por Gutiérrez, Camacho y Martínez (2007) mediante listas oficiales suministrados por las instituciones educativas y generación de números al azar, distribuidos así: 304 estudiantes en los cuales se configuró un nivel de rendimiento académico bajo en comparación con los demás alumnos y 304 estudiantes en los que las notas académicas del período escolar con corte al 30 de marzo de 2016 denotaron buen rendimiento académico. La caracterización y composición de la muestra fue por oportunidad ya que el enfoque del rendimiento académico de los estudiantes debía prevalecer para ser seleccionados dentro de alguno de los grupos. La selección detallada de la muestra se ilustra en la Tabla 1.

Los alumnos, tras ser clasificados con base al rendimiento académico (alto versus bajo) cumplimentaron un cuestionario enmarcado en la percepción del apoyo académico recibido por parte de los padres en situaciones como refuerzo positivo, desarrollo de tareas, asesoría para resolución de conflictos, cercanía paterna con el centro escolar, fijación de normas conducentes al cumplimiento de los deberes académicos, comunicación padres hijos y relaciones de afecto familiar. 
Tabla 1. Composición de la muestra por grado escolar, género, edad y rendimiento académico

\begin{tabular}{|c|c|c|c|c|c|}
\hline \multirow{2}{*}{$\begin{array}{l}\text { Grado en } \\
\text { Secundaria }\end{array}$} & \multirow{2}{*}{$\begin{array}{c}\text { Rango de } \\
\text { edades }\end{array}$} & \multicolumn{2}{|c|}{ Rendimiento académico alto } & \multicolumn{2}{|c|}{ Rendimiento académico bajo } \\
\hline & & mujeres & hombres & mujeres & hombres \\
\hline Grado sexto & $11-12$ & 38 & 38 & 38 & 38 \\
\hline Grado séptimo & $12-13$ & 38 & 38 & 38 & 38 \\
\hline Grado octavo & $13-14$ & 38 & 38 & 38 & 38 \\
\hline Grado noveno & $14-16$ & 38 & 38 & 38 & 38 \\
\hline Total estudiantes & & 152 & 152 & 152 & 152 \\
\hline Total muestra & & & & & 608 \\
\hline
\end{tabular}

Fuente: Propia

En el estudio se definen como variables a analizar las siguientes: a) Nivel de apoyo de los padres en el proceso escolar de los hijos; b) Cercanía de los padres con el centro escolar; c) Acompañamiento de los padres en la realización de las tareas; d) Fijación de normas escolares en la casa; e) Motivación y expectativas de los padres en la escolaridad de los hijos; f) Comunicación entre padres e hijos. Variables que se miden mediante instrumento adaptado de escalas usadas en investigaciones precedentes con enfoque similar al estudio en los cuales se concluye la incidencia del apoyo académico parental en el rendimiento estudiantil. De igual modo se da cabida a la indagación sobre caracterización sociodemográfica del estudiante. Para ello, en la primera parte del cuestionario se recoge información sobre el sexo y la edad del educando, personas con quien vive (padres, tíos, abuelos,..) y el nivel de estudios de los padres, dato que se confronta con las fichas académicas.

Para medir la percepción de los alumnos conformantes de la muestra, en cuanto al apoyo académico brindado por los padres y establecer la posible correlación con el rendimiento escolar, se analizaron cuatro instrumentos usados por investigadores precedentes, generando una escala adaptada a las necesidades del presente estudio, compuesta por 35 ítems nombrándola como Escala de Percepción del Apoyo Académico Parental en Tuluá bajo la sigla EPAPTUL. Las escalas analizadas para la definición final del instrumento y que hacen parte de la base de datos PsycTEST fueron las siguientes: a) Medida de Refuerzo positivo. Con esta encuesta, Li (2014) busca evaluar el grado de experiencia en el refuerzo positivo de los padres bajo la visión de los adolescentes. b) Medición de desarrollo de tareas con la ayuda de los padres. Diseñada por Dumont (2012) está dirigido a los estudiantes con el propósito de medir el nivel de acompañamiento de los padres hacia los hijos en la realización de las tareas escolares en el hogar. Mide tres enfoques: el nivel de acompañamiento, el conflicto que produce hacer tareas y la competencia de los padres para realizar las tareas. c) Escala de percepción del apoyo académico de los padres. Escala diseñada por Chen (2008) tiene como objetivo medir la auto-percepción de los estudiantes del grado de apoyo académico proporcionado por los padres. d) Percepción del estudiante respecto al 
involucramiento parental en la realización de tareas. Escala original de Núñez, Suárez, Rosário, Vallejo, Valle y Epstein (2015) con la que a una muestra de 1.683 estudiantes españoles matriculados en educación secundaria se midió la percepción que tenían del apoyo parental en la realización de las tareas, seguimiento y control de las mismas y su correspondiente correlación con el rendimiento académico.

En la tabla 2 se registran los 35 reactivos de la escala definitiva y la fuente origen indicando de igual modo si el texto se conservó como en su primera versión o si fue adaptado a las necesidades del estudio actual. La indagación realizada a partir de los ítems proyectados permite obtener información relacionada a la percepción que tengan los estudiantes sobre el apoyo académico que les brindan los padres en diferentes aspectos relacionados al desempeño y entorno escolar. En cada uno de los reactivos, los estudiantes pueden dar respuesta en una escala de 1 a 5: 1. Completamente en desacuerdo; 2. En desacuerdo; 3. Neutral. Ni en acuerdo, ni en desacuerdo; 4. De acuerdo; 5. Completamente en desacuerdo; concretando de este modo el propósito de la investigación al medir las variables definidas y la correlación que se presenta entre el rendimiento académico y el apoyo de los padres en el proceso educativo de los estudiantes encuestados. Para la puesta en marcha del instrumento, se contó de forma previa con el consentimiento por escrito de los padres de los estudiantes que hacían parte de la muestra, docentes y directivos de las instituciones educativas focalizadas, llevando a cabo el procedimiento de recolección de datos durante una sesión de clases de 60 minutos.

Tabla 2. Origen de ítems para instrumento de Investigación EPAPTUL

\begin{tabular}{|c|c|c|c|}
\hline Ítem estudiado & $\begin{array}{c}\text { Instrumento } \\
\text { Origen } \\
\end{array}$ & $\begin{array}{l}\text { Fuente } \\
\text { Origen }\end{array}$ & $\begin{array}{c}\text { Característica } \\
\text { Texto }\end{array}$ \\
\hline Mi madre me anima y motiva para el cumplimiento de mis deberes escolares. & 1 & $\mathrm{Li}$ & Texto adaptado \\
\hline Mi padre me anima y motiva para el cumplimiento de mis deberes escolares. & 1 & $\mathrm{Li}$ & Texto adaptado \\
\hline Mis padres me ayudan con las tareas si tengo dificultades. & 2 & Dumont & Igual al original \\
\hline Las tareas frecuentemente causan discusiones en mi familia. & 2 & Dumont & Igual al original \\
\hline Cuando mis padres me explican cosas a mí, les entiendo mejor que al profesor. & 2 & Dumont & Igual al original \\
\hline Mis padres saben casi todo lo que yo aprendo en la escuela. & 2 & Dumont & Igual al original \\
\hline $\begin{array}{l}\text { Hablo frecuentemente con mis padres sobre mis problemas escolares y me ayudan a } \\
\text { encontrar la forma de resolverlos. }\end{array}$ & 3 & Chen & Texto adaptado \\
\hline Mis padres están pendientes de que me vaya bien en la escuela. & 3 & Chen & Texto adaptado \\
\hline Mis padres tienen altas expectativas de que me vaya bien en el colegio. & 3 & Chen & Igual al original \\
\hline Cuando me va bien en los exámenes mis padres me elogian. & 3 & Chen & Igual al original \\
\hline
\end{tabular}


Cuando no me va bien en un examen, mis padres me exhortan para estudiar más.

Siento una gran presión de mis padres, para que me vaya bien en el colegio.

Mis padres han hablado conmigo sobre mis planes de estudio y de trabajo para el futuro.

Mis padres quieren hacer de mí el mejor de la escuela.

Mis padres influencian en mi comportamiento

Mis padres no se dan cuenta si yo voy diariamente al colegio ni preguntan por mi desempeño en la escuela.

Mis padres nunca han hablado con mis profesores sobre mi proceso escolar.

Mis padres me compran ellos mismos los materiales que requiero.

Mis padres no asisten a las reuniones informativas escolares ni participan en actividades de la escuela.

Mis padres algunas veces me preguntan por tareas.

Mis padres no tienen tiempo para ayudarme con tareas.

Mis padres no tienen suficiente conocimiento para ayudarme en las tareas.

Mis padres están seguros que paso la mayor parte del tiempo haciendo tareas.

Mis padres estudian mi boletín de notas cuidadosamente

Mis padres de vez en cuando me preguntan sobre mis notas académicas.

Mis padres están seguros de que yo sé cómo realizar las tareas.

Mis padres me ayudan para hacer las tareas bien.

Mis padres no me colaboran con los asuntos escolares.

Mis padres me colaboran con asuntos escolares en todo momento.

Si mis padres atendieran más mis asuntos escolares, a mí me iría mejor en el colegio

Mis padres están muy pendientes de si hago siempre todas las tareas.

Creo que mis padres no se enteran de si hago o no las tareas

Mis padres le dan mucha importancia a mi trabajo con las tareas y me fijan normas para la realización de las mismas.

Para mis padres es muy importante que les comparta mis experiencias en la escuela. Mis padres comparten conmigo diferentes espacios en los que se pueden aprender y recordar cosas de la escuela.
Chen Igual al original

Chen Igual al original

Chen Texto adaptado

Chen Igual al original

Chen Igual al original

Chen Texto adaptado

Chen Igual al origina

Chen Texto adaptado

Chen Texto adaptado

Chen Igual al original

Chen Igual al original

Chen Igual al original

Chen Igual al original

Chen Texto adaptado

Chen Igual al original

Chen Igual al original

Chen Igual al original

Chen Texto adaptado

Chen Texto adaptado

Chen Texto adaptado

Núñez Igual al original

Núñez Igual al original

Núñez Texto adaptado

Núñez Texto adaptado

Núñez Texto adaptado

Nota: Instrumentos origen: 1. Li (2014). Medida de Refuerzo Positivo; 2. Dumont (2012). Medición de desarrollo de tareas con la ayuda de los padres; 3. Chen (2005). Escala de percepción del apoyo académico de los padres; 4 . Núñez (2015). Percepción del estudiante en el involucramiento parental en las tareas. 
Para Pantoja (2015) la fiabilidad representa la estabilidad de la medida y conlleva a que se puede tomar como fiable una prueba si al ejecutarla en dos aplicaciones diferentes las puntuaciones son similares. Para medir la fiabilidad de una escala se pueden tomar como referentes tres tipos de coeficientes: el coeficiente Testretest, el coeficiente dos mitades planteado por Spearman-Brown y el coeficiente Alfa de Cronbach o modelo de consistencia interna. En registros de Hernández, Fernández y Baptista (2011), los coeficientes de confiabilidad deben ser interpretados según la aproximación a cero o a uno, teniendo como referentes secuenciales en resultados los niveles descritos a continuación: 0,25 indica baja confiabilidad; 0,50 fiabilidad regular o media; 0,75 confiabilidad aceptable y en los casos mayores a 0,90 fiabilidad elevada.

Una vez generada la escala EPAPTUL, se aplicó a la misma análisis de fiabilidad mediante alfa de Cronbach con un resultado de 0,914 indicando en la Tabla 3 los cálculos estadísticos generados por el programa SPSS 22. El resultado 0,914 permite referir que el nivel de confiabilidad de la escala EPAPTUL es de fiabilidad elevada, al igual que las escalas que las originaron en cuyos cálculos se registran valores superiores a 0,90 respecto al mismo indicador, denotando al igual que para la versión derivada una confiabilidad elevada en términos estadísticos.

Tabla 3. Coeficiente general de Fiabilidad de la escala EPAPTUL

\begin{tabular}{cc}
\hline \multicolumn{2}{c}{ Estadísticas de fiabilidad } \\
\hline Alfa de Cronbach & $\mathrm{N}$ de \\
& elementos \\
$\mathbf{9 1 4}$ & 35 \\
\hline
\end{tabular}

La validez, en orientaciones de Pantoja (2015) indica el grado en el que el texto de una escala o cuestionario mide lo que dice medir. La validez se obtiene de dos formas: validando el contenido y validando el constructo. La validación del contenido, en precisiones de Hernández, Fernández y Baptista (2011) se debe llevar a cabo por medio de opiniones de expertos que desde su conocimiento avalen los reactivos de la escala, la complementen, sugieran modificaciones o inclusiones y den visto bueno del formato a usar. En cuanto a la validez de constructo, que se refiere a la evaluación de la interdependencia en los reactivos propuestos se genera mediante la técnica de análisis factorial la cual se calcula con el programa SPSS 22.

Respecto a la validez de contenido del presente estudio, se contó con el apoyo de dos expertas investigadoras a nivel educativo: Dra. Natalia Suárez Fernández quien se desempeña como profesora del área de psicología evolutiva en la Universidad de Oviedo y Dra. Gloria Rojas Ruiz, profesora de didáctica y organización escolar en la Universidad de Granada. En opinión de Suárez (2016) los reactivos contenidos en la escala de la investigación son apropiados haciendo referencia en la revisión a que dos de las afirmaciones estaban repetidas, situación que se mejoró en el texto definitivo aplicado a la muestra. Para Rojas (2016) en términos generales, la escala EPAPTUL está redactada de tal forma que cumple con el objetivo general propuesto, permitiendo 
una vez se tengan los resultados analizar el cumplimiento de la hipótesis de investigación.

Para la validez de constructo se aplicó técnica de análisis factorial mediante las pruebas KMO y esfericidad de Bartlett en el programa SPSS 22, registrando los resultados en la tabla 4 en los cuales se observa interdependencia en los 35 reactivos componentes de la escala EPAPTUL.

Tabla 4. Prueba de KMO y Bartlett Escala EPAPTUL

\begin{tabular}{lll}
\hline \multicolumn{2}{l}{ Medida Kaiser-Meyer-Olkin de adecuación de muestreo } &, 952 \\
\hline Prueba de esfericidad de Bartlett & Aprox. Chi-cuadrado & 46795,017 \\
& gl & 595 \\
& Sig. & 0,000 \\
\hline
\end{tabular}

Fuente: Propia

La medida KMO con un resultado de 0,952 indica una relación alta entre las variables lo que conlleva que el planteamiento de los reactivos han sido bien direccionados, considerándose la escala a usar dentro de las categorías de muy bueno respecto a la medida indicada, la cual se expresa en la siguiente forma: $K M O \geq 0,9$ test muy bueno; $\mathrm{KMO} \geq 0,8$ notables; $\mathrm{KMO} \geq 0,7$ medianos; $\mathrm{KMO} \geq 0,6$ bajo; $\mathrm{KMO}<0,5$ muy bajo. Los coeficientes de correlación de las variables en el instrumento medido están altamente relacionados.

Para Benavente, Cañaveras, Martínez y Pla (2011) la prueba de esfericidad de Bartlett evalúa si es posible aplicar el análisis factorial para la validez del constructo. Cuando el resultado del componente Sig es mayor a 0,05 no se puede aplicar la técnica rechazando la hipótesis nula $\mathrm{H}_{0}$ y si es menor a 0,05 se puede aplicar la técnica aceptando $\mathrm{H}_{0}$. En el instrumento de investigación, la prueba de esfericidad tiene como resultado para el componente Sig 0,000 por lo cual la técnica análisis factorial se puede aplicar dando continuidad a los cálculos de la técnica.

Una vez finalizado el trabajo de campo se analizaron los datos obtenidos por medio de la valoración propia del investigador y de los referentes teóricos que se tuvieron en cuenta para que al momento de compararlos surjan las conclusiones propias del estudio y de esta forma consolidar una estrategia que a nivel institucional permita mejorar el rendimiento académico y sirva de referente en casos similares a los estudiados. Para el análisis de los resultados se crearon categorías diferenciadas para cada grupo objetivo: niños, niñas, periodicidad de acompañamiento familiar, nivel de preparación académica de los padres, entre otros. En los análisis se empleó el programa estadístico SPSS V. 22, tanto para los análisis descriptivos como para las comparaciones entre los alumnos con un buen y bajo rendimiento con el fin de determinar el peso que dichas variables familiares tuvieran sobre el rendimiento. Al finalizar el estudio se espera comparar en ambos grupos de sujetos, los diferentes 
aspectos del apoyo académico que reciben de parte de los padres; así como determinar estrategias que los programas de asesoramiento escolar deberían contemplar de cara a optimizar el rendimiento de los estudiantes a partir del acercamiento de los padres al entorno escolar.

\section{Resultados}

Los resultados se presentan mediante análisis descriptivo, técnica que para Pantoja (2015) es la más recomendada en investigaciones con enfoque educativo, registrando de forma detallada las situaciones encontradas en las respuestas condensadas por los 608 estudiantes conformantes de la muestra. Respecto a las edades de los alumnos consultados, la edad promedio es de 12,68 años con desviación estándar de 1,4468 y varianza de 2,093. Datos estadísticos que reflejan la consistencia de la muestra analizada.

Un 9,5\% de los encuestados manifestó ser hijo único (58 alumnos). Un 13,4\% tiene un solo hermano (82 alumnos). 69,6\% tienen dos hermanos (423 alumnos). 7,4\% conviven con más de dos hermanos (45 alumnos). En cuanto a la convivencia con el padre o la madre un 10,4\% (63) viven sólo con la mamá; 7,1\% (43) sólo con el papá; 75,8\% (461) viven con ambos padres; $3,3 \%$ (20) viven con los abuelos; 2,1\% (13) con tíos y sólo un $1,3 \%$ (8) con personas diferentes a la familia. En términos de rendimiento académico, la convivencia de los estudiantes con los padres y la cantidad de hermanos presentes en el hogar se registra en las tablas 5 y 6 .

Tabla 5. Personas con quien viven los estudiantes

\begin{tabular}{lcrrrrr}
\hline \multicolumn{1}{c}{$\begin{array}{c}\text { Personas con quien vive el } \\
\text { estudiante }\end{array}$} & $\begin{array}{c}\text { Buen Rendimiento } \\
\text { académico }\end{array}$ & \multicolumn{3}{c}{$\begin{array}{c}\text { Bajo Rendimiento } \\
\text { académico }\end{array}$} & Total \\
\hline Sólo con mamá & 34 & $11 \%$ & 29 & $10 \%$ & 63 & $10,4 \%$ \\
Sólo con papá & 20 & $7 \%$ & 23 & $8 \%$ & 43 & $7,1 \%$ \\
Con papá y mamá & 238 & $78 \%$ & 223 & $73 \%$ & 461 & $75,8 \%$ \\
Con abuelo o abuela & 8 & $3 \%$ & 12 & $4 \%$ & 20 & $3,3 \%$ \\
Con tíos & 3 & $1 \%$ & 10 & $3 \%$ & 13 & $2,1 \%$ \\
Con otros no familiares & 1 & $0 \%$ & 7 & $2 \%$ & 8 & $1,3 \%$ \\
Totales & $\mathbf{3 0 4}$ & $\mathbf{1 0 0 \%}$ & $\mathbf{3 0 4}$ & $\mathbf{1 0 0 \%}$ & $\mathbf{6 0 8}$ & $\mathbf{1 0 0 , 0 \%}$ \\
\hline
\end{tabular}

Fuente: Propia

Tabla 6. Cantidad de Hermanos

\begin{tabular}{lrrrrrr}
\hline & Buen Rendimiento & Bajo Rendimiento & \multicolumn{2}{c}{ Totales } \\
\hline Ninguno & 30 & $10 \%$ & 28 & $9 \%$ & 58 & $9,5 \%$ \\
Uno & 49 & $16 \%$ & 33 & $11 \%$ & 82 & $13,5 \%$ \\
Dos & 197 & $65 \%$ & 226 & $74 \%$ & 423 & $69,6 \%$ \\
Más de dos & 28 & $9 \%$ & 17 & $6 \%$ & 45 & $7,4 \%$ \\
Totales & $\mathbf{3 0 4}$ & $\mathbf{1 0 0 \%}$ & $\mathbf{3 0 4}$ & $\mathbf{1 0 0 \%}$ & $\mathbf{6 0 8}$ & $\mathbf{1 0 0 , 0 \%}$ \\
\hline
\end{tabular}

Fuente: Propia 
A la pregunta si se sentían motivados por la mamá en el proceso escolar el 100\% de los encuestados con buen rendimiento académico manifestó estar de acuerdo (145 estudiantes) y completamente de acuerdo (159 estudiantes); mientras que el 100\% de la muestra con bajo rendimiento académico manifestó estar en desacuerdo (149 estudiantes) y completamente en desacuerdo (155 estudiantes). En respuesta al reactivo sobre la motivación del padre en la vida escolar de los hijos, el $77.4 \%$ de los encuestados con buen rendimiento académico (235 alumnos) manifestó estar completamente de acuerdo; mientras que un 22,6\% (69 alumnos) contestaron estar de acuerdo. En el grupo de bajo rendimiento un 80,2\% (244 alumnos) estuvieron completamente en desacuerdo y un 19,8\% (60 alumnos) manifestaron estar en desacuerdo.

Respecto a las expectativas de los padres para que a los niños les vaya bien en el colegio y en la proyección que visionan luego de la etapa escolar el grupo de buen rendimiento académico manifestó en un $100 \%$ percibir buenas expectativas; mientras que el grupo de bajo rendimiento dejó resultados de 62,5\% (190) estando completamente en desacuerdo; $23,7 \%$ (72) no estuvieron de acuerdo y un $13,8 \%$ (42) estuvieron indiferentes al reactivo.

En el reactivo "mis padres me ayudan con la tarea si yo se los pido" el 74,4\% con buen rendimiento están de acuerdo (226 estudiantes); paralelo a la posición en desacuerdo del 79,2\% del grupo de bajo rendimiento (241 estudiantes). Si en la elaboración de las tareas los padres perciben dificultad de los hijos al momento de realizarlas, un $78 \%$ de los niños del primer grupo responde que los padres les ayudan de forma voluntaria (237) y un $72 \%$ del segundo grupo responde que sus padres no les colaboran en la dificultad al momento de hacer las tareas (219).

En el ítem "si mis padres atendieran más mis asuntos escolares, a mí me iría mejor en el colegio" el $97 \%$ de los alumnos segmentados en bajo rendimiento académico (295) perciben como positiva la afirmación estando de acuerdo y completamente de acuerdo con el ítem, mientras que a sólo un 3\% (9) les es indiferente la situación planteada. Se halla el índice de Pearson para correlacionar la variable citada con el rendimiento académico obteniendo un resultado de 0,862 lo que indica una correlación casi perfecta entre las dos variables, interpretando el resultado de forma positiva para el rendimiento académico en la situación de que si los padres atienden con mayor tiempo y dedicación los asuntos escolares de los hijos, los resultados en rendimiento serían mejores.

En el reactivo "mis padres me colaboran con asuntos escolares en todo momento" 297 alumnos del primer grupo encuestado $(97,7 \%)$ están de acuerdo y relacionan el rendimiento con la colaboración de los padres; mientras que 242 del segundo grupo están en desacuerdo $(79,6 \%)$. Datos que se registran en la tabla 7. 
Tabla 7. Colaboración permanente de los padres en asuntos escolares

\begin{tabular}{llrr}
\multicolumn{1}{c}{ Rendimiento } & \multicolumn{1}{c}{ Escala } & Frecuencia & Porcentaje \\
\hline & Neutral ni en acuerdo ni en desacuerdo & 7 & 2,3 \\
Buen Rendimiento académico & De acuerdo & 226 & 74,3 \\
& Completamente de acuerdo & 71 & 23,4 \\
& Total & $\mathbf{3 0 4}$ & $\mathbf{1 0 0 , 0}$ \\
\hline & Completamente en desacuerdo & 196 & 64,5 \\
Bajo Rendimiento académico & En desacuerdo & 46 & 15,1 \\
& Neutral ni en acuerdo ni en desacuerdo & 62 & 20,4 \\
& Total & $\mathbf{3 0 4}$ & $\mathbf{1 0 0 , 0}$ \\
\hline
\end{tabular}

Fuente: Propia

En el reactivo "mis padres estudian mi boletín de notas cuidadosamente" el $70,7 \%$ (215) de estudiantes con buen rendimiento manifestaron estar totalmente de acuerdo y el 29,3\% (89) estuvieron de acuerdo. Yuxtapuesto a ello y en los mismos porcentajes el grupo de bajo rendimiento académico estuvo totalmente en desacuerdo en un $70,7 \%$ y en desacuerdo un $29,3 \%$. Analizada la relación entre este reactivo y el rendimiento académico por medio de la correlación de Pearson, se obtienen los resultados contenidos en la tabla 8 con la cual se puede observar una casi perfecta correlación de -0,966 interpretando que entre menos tiempo dediquen los padres a estudiar el boletín de notas menor será el rendimiento académico.

Tabla 8. Correlación de Pearson para variable estudio boletín de notas

\begin{tabular}{llrr} 
& & \multicolumn{1}{c}{$\begin{array}{c}\text { Mendimiento } \\
\text { académico }\end{array}$} & $\begin{array}{c}\text { Mis padres estudian mi } \\
\text { boletín de notas } \\
\text { cuidadosamente }\end{array}$ \\
\hline \multirow{2}{*}{ Rendimiento académico } & Correlación de Pearson & 1 &,$- 966^{* *}$ \\
\cline { 2 - 4 } & Sig. (bilateral) & 608 & 0,000 \\
\cline { 2 - 4 } $\begin{array}{l}\text { Mis padres estudian mi boletín } \\
\text { de notas cuidadosamente }\end{array}$ & $\mathrm{N}$ Correlación de Pearson &,$- 966^{* *}$ & 608 \\
\cline { 2 - 4 } & Sig. (bilateral) & 0,000 & 1 \\
\cline { 2 - 4 } & $\mathrm{N}$ & 608 & 608 \\
\hline
\end{tabular}

Fuente: Propia

En la tabla 9 se presentan los resultados porcentuales del ítem "mis padres no tienen suficiente conocimiento para ayudarme en las tareas" en el que se puede observar que el $100 \%$ de los encuestados del grupo de bajo rendimiento académico manifiestan estar de acuerdo con el referente citado. Si se comparan estos resultados con el nivel académico de los padres registrados en la Figura 1, se advierte que un 10,20\% (62 padres) cuentan con estudios universitarios y son padres de estudiantes con buen 
rendimiento; en contraste a sólo un $0,82 \%$ (5 padres) con estudios universitarios en el grupo de bajo rendimiento.

Tabla 9. Conocimiento de los padres para brindar apoyo en realización de tareas.

\begin{tabular}{lcccccc} 
& $\begin{array}{c}\text { Completamente } \\
\text { en desacuerdo }\end{array}$ & En desacuerdo & $\begin{array}{c}\text { Neutral ni en } \\
\text { acuerdo ni en } \\
\text { desacuerdo }\end{array}$ & $\begin{array}{c}\text { De acuerdo } \\
\text { Completamente } \\
\text { de acuerdo }\end{array}$ \\
\hline Buen Rendimiento académico & $15,5 \%$ & $66,1 \%$ & $18,4 \%$ & 0 & 0 \\
\hline Bajo Rendimiento académico & 0 & 0 & 0 & $61,5 \%$ & $38,5 \%$ \\
\hline
\end{tabular}

Fuente: Propia

En el reactivo "Mis padres no asisten a las reuniones informativas ni participan en actividades de la escuela" el 100\% del grupo de estudiantes con buen rendimiento académico manifestó completo desacuerdo $(68,4 \%)$ y desacuerdo $(31,6 \%)$ con la situación planteada, infiriendo de la respuesta el sentido contrario a la asistencia de los padres y la continua participación en actividades escolares. En el grupo de alumnos con bajo rendimiento los resultados son favorables a la negación planteada. Un 67,1\% manifestó estar de acuerdo (204 alumnos); 26\% totalmente de acuerdo (79) y para un $6,9 \%$ (21) les fue indiferente el ítem.

Figura 1. Nivel educativo de los Padres

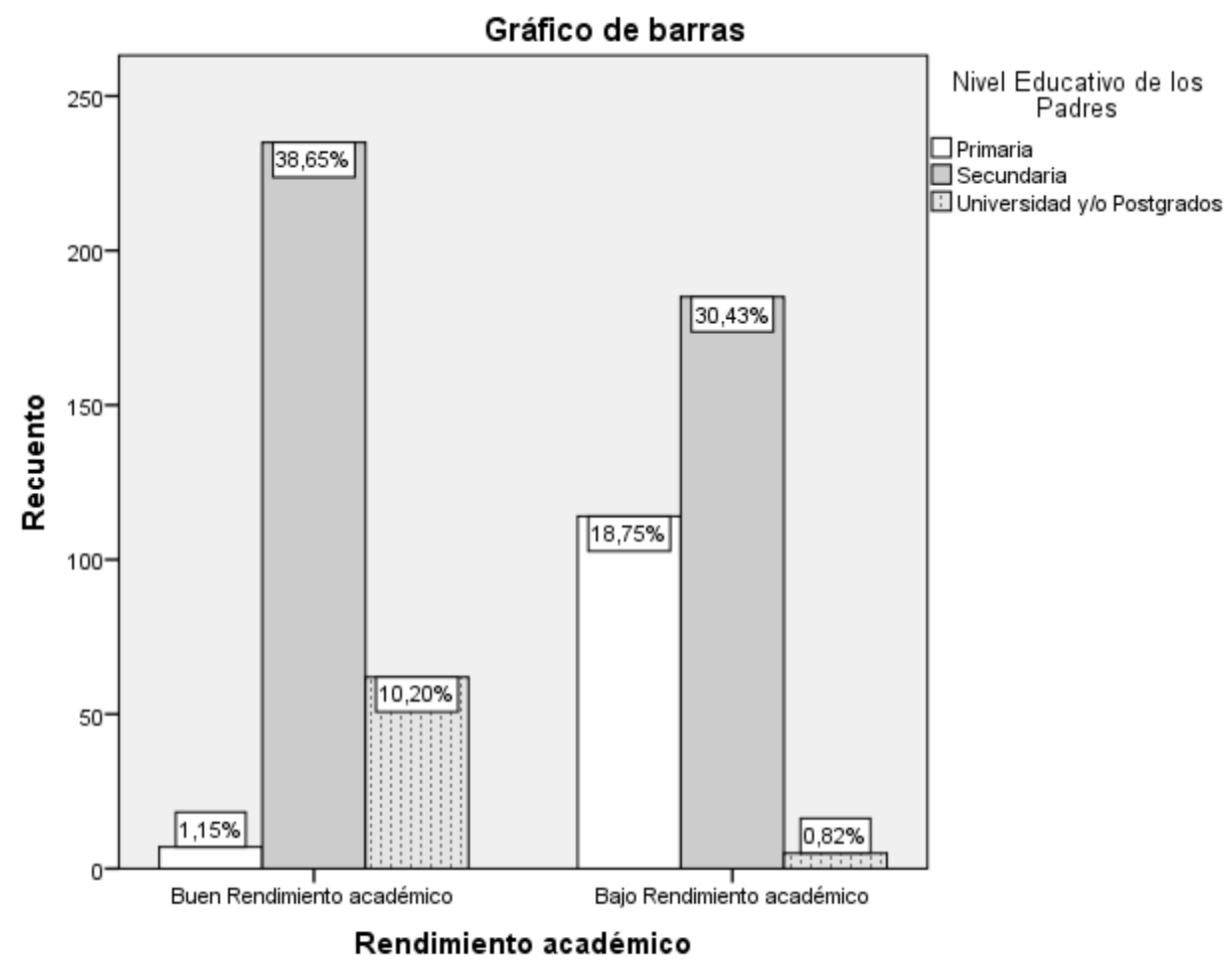


Paralelo a los resultados anteriores, en el escenario esbozado de que los hijos hablan frecuentemente con los padres sobre problemas escolares y les ayudan a encontrar la forma de resolverlos, el $100 \%$ de estudiantes con buen rendimiento académico manifestó estar de acuerdo $(33,8 \%)$ y totalmente de acuerdo (66,2\%). En el grupo de bajo rendimiento los resultados son negativos al ítem planteado con un 3,9\% (12 alumnos) que no están de acuerdo ni en desacuerdo; un 51,4\% (156 alumnos) están en desacuerdo y 44,7\% (136 alumnos) responden completamente en desacuerdo.

En el ítem "Mis padres le dan mucha importancia a mi trabajo con las tareas y me fijan normas para la realización de las mismas" el 84,8\%, esto es 258 estudiantes, con buen rendimiento académico manifestó estar totalmente de acuerdo y el saldo restante estuvo de acuerdo. En contraste el 76,6\% (233) del grupo de bajo rendimiento respondió estar totalmente en desacuerdo, frente a un 23,4\% (71 alumnos) que estuvieron en desacuerdo. Usando el indicador Rho de Spearman se correlaciona la variable citada con la variable de rendimiento académico, obteniendo un resultado casi perfecto de -0,910 lo cual se interpreta que en la medida que los padres no le den importancia a las tareas ni fijen normas para la realización de los deberes por parte de los hijos, el rendimiento baja.

\section{Discusión y conclusiones}

El artículo hace posible que la investigación descrita sea parte del conjunto de estudios que sobre el tema de rendimiento académico en adolescentes existen a la fecha, fomentando de esta forma el ánimo de los docentes y de las familias para que desde los propios contextos de la escuela o del hogar se consoliden estrategias de mejoramiento con las que se procure que los padres vivencien de cerca la escolaridad de los hijos aportándoles el apoyo necesario para lograr un acertado aprendizaje y resultados satisfactorios en el proceso de formación integral del estudiante. El artículo es coherente con las investigaciones referidas en la introducción en cuanto a las conclusiones que correlacionan de forma directa el apoyo académico parental con el rendimiento escolar. A mayor apoyo de los padres, mejor es el resultado en el rendimiento de los adolescentes; por el contrario, a menor apoyo percibido menor es el rendimiento obtenido, lo cual es coherente con las conclusiones de investigaciones realizadas por Erazo (2010) y por Waqas, Fatima, Sohail, Saleem y Khan (2013).

En la variable "número de hermanos" se observa que la medida de dos hermanos difiere en un $9 \%$ entre uno y otro grupo, siendo mayor en los de bajo rendimiento, concluyendo que a mayor cantidad de hermanos, menor va a ser el tiempo que los padres tengan para dedicarle a uno solo. Respecto a la convivencia con uno solo de los padres, con los dos, con abuelos, tíos o particulares la tendencia de los resultados muestra que quienes viven en el seno de una familia tradicional están mayormente agrupados en la característica de buen rendimiento académico, siendo superior en un $5 \%$ frente al grupo que presenta rezago en el rendimiento. 
Tanto la motivación del padre como la de la madre son percibidas en gran proporción en los estudiantes con buen rendimiento. Motivación que es escasa en los alumnos agrupados con bajo desempeño. En el primer grupo la motivación del padre es del $77,4 \%$ en la escala completamente de acuerdo y la de la madre en un $52,30 \%$ concluyendo que si el alumno observa mayor motivación del padre en aspectos académicos los resultados escolares se fortalecen. En el grupo de bajo rendimiento, la motivación tanto del padre como de la madre es escasa. En cuanto a la vida futura de los estudiantes luego de terminar el ciclo secundario, las expectativas de los padres son percibidas como excelentes en el grupo de buen rendimiento y deficientes en el grupo de bajo rendimiento. Similares conclusiones en cuanto a la percepción se encuentran en investigaciones realizadas por Hoover-Dempsey (2001), Giordano (2003), Chen (2008) y Dumont, Trautwein, Nagy y Nagengast (2013).

En los reactivos relacionados a la variable sobre acompañamiento en la realización de tareas, al igual que para los autores Núñez, Suárez, Rosário, Vallejo, Valle y Esptein (2015) así como para Faires, Nichols y Rickelman (2000) se concluye que el acompañamiento en estudiantes con buen rendimiento académico es óptimo dándose de forma voluntaria, con retroalimentación, seguimiento y ejemplificación constante de los padres hacia los hijos. En los momentos compartidos los padres tienden a ilustrar las situaciones escolares, brindando las explicaciones necesarias cuando los temas no son muy claros para ellos. En este sentido se concluye también que el nivel educativo de los padres es altamente influyente en los resultados académicos de los hijos, observando que el alcance de grados universitarios en el grupo de buen rendimiento es mayor que en el grupo de bajo rendimiento superándolo en un $10 \%$ de la muestra estudiada. Paralelo a ello, en el grupo de bajo rendimiento no hay acompañamiento alguno por parte de los padres en la realización de tareas y hay poco conocimiento de los mismos en los contenidos educativos de los hijos, extendiendo este poco o nulo alcance en términos de orientación y comunicación; aspectos en los cuales se concluye que los adolescentes con buen desempeño presentan características de comunicación y orientación fluida, abierta y constante con los padres.

Para finalizar se concluye que los estudiantes con bajo rendimiento académico perciben que los resultados en la escuela serían mejores si contaran con una debida y completa atención paternal, centrando su percepción en momentos como acompañamiento en la realización de tareas, motivación para asistir a clases, expectativas a futuro, cercanía con los docentes y con el centro escolar, comunicación en la casa, afecto, colaboración y orientación permanente. Dentro de las limitaciones del estudio se cuentan variables propias de los estudiantes tales como el temor al sentirse investigados, cambios en su comportamiento habitual lo que puede conllevar a generar respuestas incorrectas en los reactivos propuestos y rechazo de las familias a la investigación realizada sin dar el consentimiento de llevarla a cabo. Como línea de continuidad se hace necesario fijar la influencia en el rendimiento académico de 
variables propias del estudiante tales como la depresión y los cambios constantes de humor causados por la etapa natural de la adolescencia.

La investigación realizada permite plantear como alternativas de mejoramiento del rendimiento académico, la generación de estrategias en las instituciones educativas que generen acercamiento paternal al centro escolar proponiendo actividades como bazares, conversatorios, talleres, escuelas de padres en la que participen de igual modo los hijos, conferencias, citaciones y en general abrir la posibilidad de una comunicación abierta entre padres y colegio; con el fin de que esta cercanía se extienda al ámbito familiar, propiciando el apoyo académico que los niños necesitan en la casa para fortalecer el proceso académico.

\section{Referencias}

Bas, E. y Pérez M.V. (2010). Familia y sociedad contemporánea. Educación Siglo XXI, 28(1), 10-15.

Benavente, D., Cañaveras, J., Martínez, J. y Pla, C. (2011). Análisis multivariante con SPSS. Reducción de datos: Análisis de Componentes Principales y Factorial. Recuperado de http://web.ua.es/es/lpa/docencia/practicas-analisis-exploratorio-dedatos-con-spss/practica-5-analisis-multivariante-con-spss-reduccion-de-datosanalisis-de-componentes-principales-y-factorial.html

Bodensiek, A. (2010). Estudio sobre los factores que influyen en el rendimiento escolar. Bogotá: Secretaría de Educación Municipal.

Caicedo G., y Cepeda, E. (2007). Factores asociados a la calidad de la educación. Revista Iberoamericana de Educación, 15(43), 65-89.

Chen, J. (2008). Grade level differences: relations of parental, teacher and peer support to academic engagement and achievement among Hong Kong students. School Psychology International, 29, 183-198.

Desforges, C. y Abouchaar, A. (2003). The impact of parental involvement, parental support and family education on pupil achievements and adjustments: A literature review. Nottingham: Queen's Printer.

Dumont, H., Trautwein, U., Ludtke, O., Neumann, M., Niggli, A. y Schnyder, I. (2012). Parental Homework Involvement Measure. PsycTESTS. Recuperado de http://psycnet.apa.org/?\&fa=main.doiLanding\&doi=10.1037/t21365-000

Dumont, H., Trautwein, U., Nagy, G. y Nagengast, B. (2013). Quality of parental homework involvement: predictions and reciprocal relations with academic 
functioning in the reading domain. Journal of Educational Psychology, 106(1), 144-161. doi: 10.1037/a0034100.

Enríquez, C., Segura, A. y Tovar, J. (2013). Factores de riesgo asociado a bajo rendimiento en escolares de dos instituciones educativas públicas de Bogotá. Investigaciones Andina, 26(15), 654-666.

Erazo, O. (2010). El estudiante y sus problemas en la escuela. Revista de Psicología GEPU, 1(2), 40-57.

Faires, J., Nichols, W. y Rickelman, R. (2000). Effects of parental involvement in developming competent readers in first grade. Reading Psychology, 21(2), 95215.

Giordano, P.C. (2003). Relationships in adolescence. Annual Review of Sociology, 29(3), 257-281.

Gutiérrez, P., Camacho, N. y Martínez, M. (2007). Autoestima, funcionalidad familiar y rendimiento escolar en adolescentes. Aten Primaria, 39(11), 597-601.

Hernández, R., Fernández, C. y Baptista, P. (2011). Metodología de la investigación. México: Editorial Mc Graw Hill.

Hernando, A., Oliva, A., y Pertegal, M. (2012). Variables familiares y rendimiento académico en la adolescencia. Estudios de Psicología, 33(15), 51-65.

Hoover-Dempsey, K. (2001). Parental involvement in homework. Educational Psychologist, 36(4), 195-209.

Iraurgi, I., Martínez, A., Iriarte, L. y Sanz, M. (2011). Modelo cognitivo contextual del conflicto interparental y la adaptación de los hijos. Anales de Psicología, 27(2), $562-573$.

Li, S. (2014). Positive Reinforcement Measure PsycTESTS. Recuperado de http://psycnet.apa.org/?\&fa=main.doiLanding\&doi=10.1037/t36570-000.

Lozano, A. (2003). Factores personales, familiares y académicos que afectan el fracaso escolar en la Educación secundaria. Revista electrónica de investigación psicoeducativa y psicopedagógica, 1(1), 43-66.

Núñez, J., Suárez, N., Rosario, P., Vallejo, G., Valle, A. y Epstein, L. (2015). Relationships between perceived parental involvement in homework, student homework behaviors, and academic achievement: differences among elementary, junior high, and high school students. Metacognition Learning, 10, 375-406. doi: 10.1007/s11409-015-9135-5 
Núñez, J., Vallejo, G., Rosário, P., Tuero, E. y Valle, A. (2014). Student, teacher and school context variables predictin academic achievement in Biology: analysis from a multilevel perspective. Revista de Psicodidáctica, 19, 145-171.

Oubrayrie-Roussel, N. y Safont-Mottay, C. (2011). Adolescent homework management strategies and perceptions of parental involvement. International Journal about parents in education, 5 , 78-85.

Pantoja, A. (2015). Manual Básico para la realización de tesinas, tesis y trabajos de investigación. Madrid: EOS.

Peralta, J. y Espinoza, J. (2009). Estudio de la violencia intrafamiliar y su incidencia en la adaptación escolar y el desarrollo académico de los estudiantes del quinto año de básica de la escuela Aurelio Aguilar Vásquez, de Cuenca Ecuador. Revista Universidad Nacional de Ecuador, 5(1), 16-22.

Porta, E. y Laguna, J. (2008). Equidad de la educación en Guatemala. Serie de Investigaciones educativas, 4(1), 23-43.

Risso, A., Peralbo, M., y Barca, A. (2010). Cambios en las variables predictoras del rendimiento escolar en enseñanza secundaria. Psicothema Universidad de Coruña, 10(4), 790-796.

Schlee, B.M., Mullis, A.K., y Shriner, M. (2009). Parents's social and resource capital: Predictors of academic achievement during early childhood. Children and Youth Services Review, 31(5), 227-234.

UNNE. (2016). Calculadora para obtener el tamaño de una muestra. Recuperado de http://med.unne.edu.ar/biblioteca/calculos/calculadora.htm

Waqas, H., Fatima, T., Sohail, M., Saleem, M. y Khan, M. (2013). Parental Involvement and Academic Achievement: A study on secondary school students of Lahore, Pakistan. International Journal of Humanities and Social Science, 8(3), 209223.

$\mathrm{Xu}$, J. y Corno, L. (2006). Gender, family help and homework management reported by middle school students. Journal of Research in Rural Education, 21, 1-13.

Fecha de recepción: 01/09/2016

Fecha de revisión: 26/10/2016

Fecha de aceptación: 02/11/2016 